\title{
Stability Control and Simulation Analysis of Automobile Hydraulic System ESP
}

\author{
Liu Yongqiu', Liu Liping ${ }^{2}$ \\ ${ }^{1}$ Guangdong University of Science \& Technology, Dongguan, 523083, China \\ ${ }^{2}$ Changchun Vocational Institute of Technology \\ Changchun, 13000
}

\section{Keywords: Brake, Fuzzy PID Control, AMESim}

\begin{abstract}
Role car ESP hydraulic system is applied to the appropriate wheel hydraulic braking force, the implementation of active intervention of the vehicle, so that the car during braking, good stability, and therefore directly affect the effective control of the ESP braking car security. Thesis for the dynamic characteristics of cars ESP hydraulic system research, based on AMESim software to establish the ESP hydraulic system model is proposed fuzzy PID as the ESP control strategies, the use of AMESim and Simulink co-simulation results show that the method can effectively improve the car's braking stability, there are certain practical significance.
\end{abstract}

\section{Introduction}

With advances in technology to improve vehicle and road traffic, vehicle speed is increasing. In all types of accidents, due to an accident caused by a vehicle at high speed instability is gradually increased [1]. When car at high speed state of the brake, emergency steering, slippery road surfaces, etc. influence, tire lateral force will be close to adhesion limits[2], which makes cars produce oversteer or understeer problems, making the car deviates from a predetermined route, thereby traffic accidents. Therefore, to improve the lateral stability of the vehicle during braking at the limit conditions for the safety of the vehicle plays an important role. Automotive electronic stability control system (ESP) as an active safety technology that actively suppress the car oversteer or understeer tendency, improve handling stability and safety when driving cars, ESP control strategy in what is currently the research staff focus[3].

In this paper, ESP hydraulic system as the research object, based on AMESim software to build a model of the hydraulic system ESP, proposed fuzzy PID control strategy, the vehicle yaw rate and sideslip angle as the evaluation index [4][5], co-simulation in MATLAB environment, the analysis results show that the fuzzy PID control strategy can effectively solve the problem of automobile braking instability, has some practical value.

\section{Working principle of the ESP}

The basic principle of the ESP control is through the sensor and the operation logic to identify the driver operating intentions expect of automobile motion, at the same time measure and estimate the actual motion state of the car[6]. When the error between the two is greater than the set value, the control logic of the ESP system in a certain size of the longitudinal force to the corresponding control and adjustment, make the role change of the yawing moment in the car, so that the actual motion state is closer to the driver desired motions. As shown in figure 1, when. When the car appear over steering trend, the ESP by applying the brake wheel and car has turned to opposite direction yawing moment, make the car back to pilot the desired track[7]. When auto show the tendency of under steer, ESP by applying the brake at the rear wheels and automotive steering movement direction of the same yawing moment, the horizontal swing of the car. 


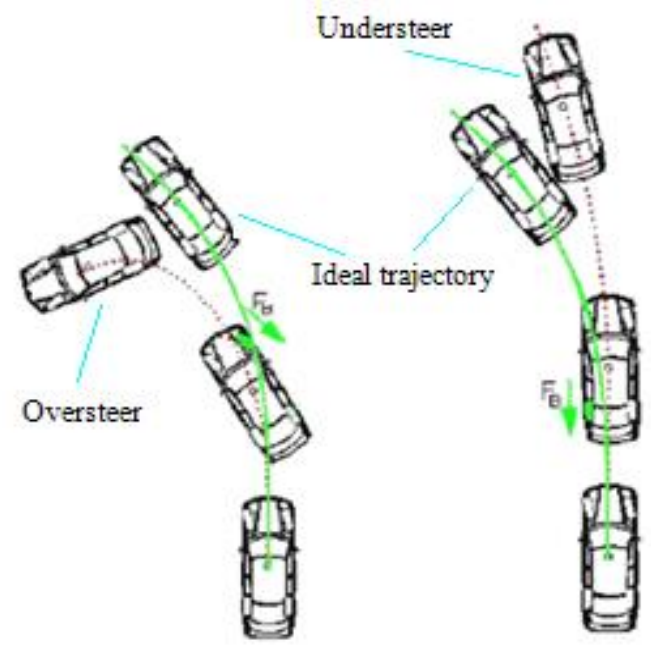

Fig.1 The control principle of the ESP

\section{Main method of stability control}

There are many ways to improve the stability of the car through the active control, methods applied at present basically has: active steering control system, vertical load control and power/force control.

1) The active steering control

Active steering control can be achieved by vehicle movement state feedback to improve the car in steering the steering stability of the linear zone, can be in a certain extent, and inhibit side-slip Angle of mass center of the car. But when the vehicle movement state into nonlinear area, through to adjust attitude will be very difficult, effect is not obvious [8].

2) Vertical load control

Vertical load control is by controlling the vertical load on the wheel to change the front axle of equivalent stiffness, so as to enhance the stability of the car. Vertical load is indirect control of vehicle stability control, the car will be required to have active suspension system, and the control effect is not obvious when the acceleration is hours.

3)Horizontal pendulum control

Horizontal pendulum control is by changing different wheel braking force/driving force to improve the stability of the vehicle active safety technology. Among them, change the way that the braking force is called active braking, applying different force through the different wheels to produce extra yawing moment. Change the way that the driver is called variable torque distribution, is to pass on the left and right driving wheels on different driving force to create change posture yawing moment.

In summary, through active braking stability control implementation, low cost, easy to implement, wide range of applications. Therefore, the use of proactive approach to vehicle stability control braking, especially under extreme tire adhesion stability control is the most appropriate, and is currently the most common method of controlling most of the stability control system.

\section{ESP hydraulic system}

\section{The composition of ESP hydraulic system}

Automobile ESP hydraulic system mainly including brake operating mechanism, brake master cylinder, the hydraulic control unit and brake components, such as concrete as shown in figure 2 . 


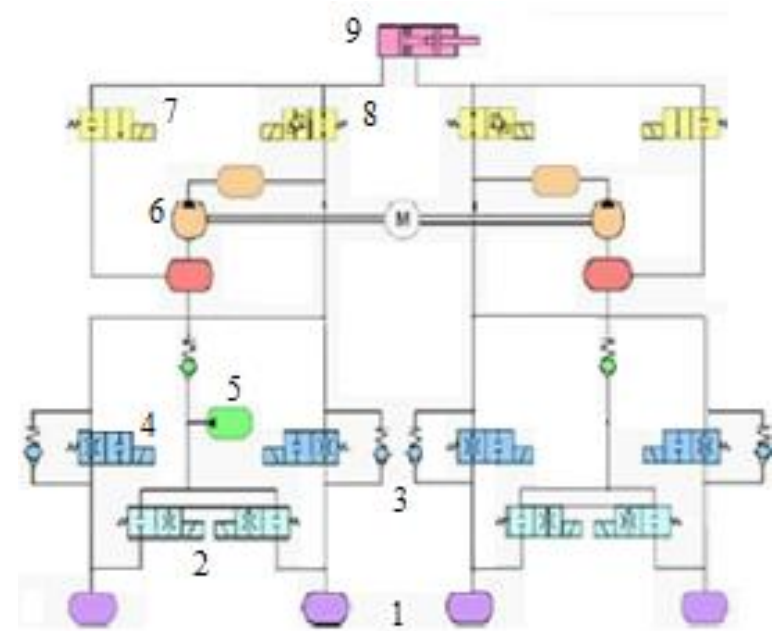

1-brake wheel cylinder; 2-pressure reducing valve

3-check valve; 4-pressurization valve;5-accumulator

6-pump; 7-Suction valve; 8-Isolation valve;9-brake master cylinder

\section{AMESim model}

Fig.2 ESP hydraulic system structure

Using AMESim hydraulic components library car ESP hydraulic system model is set up, ignore the leak in the process of modeling, liquid compression factors, and such as is shown in figure 3 .

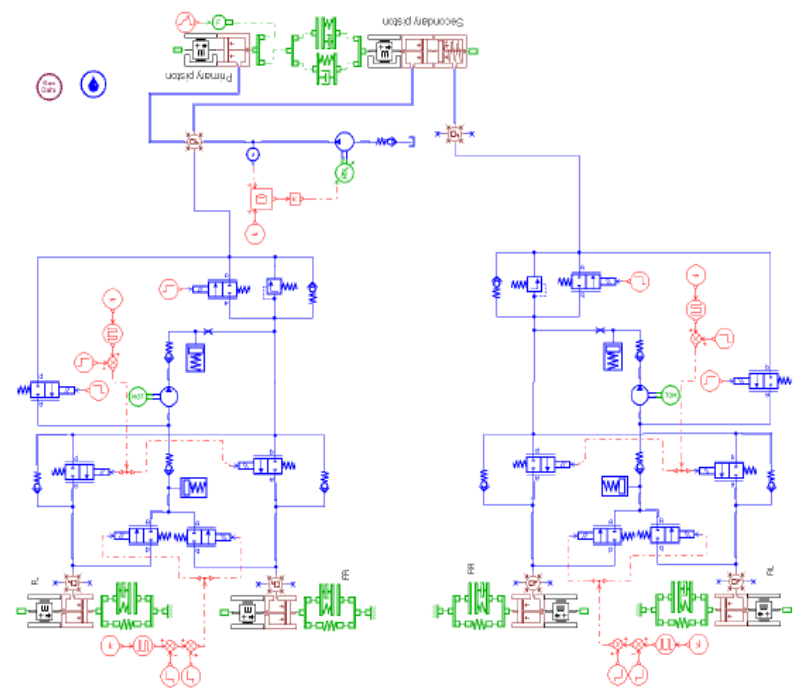

Fig.3 The hydraulic system AMESim model

\section{Fuzzy control strategy research}

\section{The fuzzy PID controller}

In order to ensure the control accuracy, in this paper, the application of fuzzy inference to ESP hydraulic control system [9], and combined with the traditional PID control strategy, we design a fuzzy PID controller; the structure is shown in figure 4.

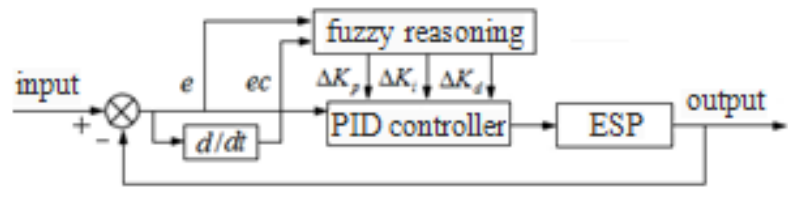

Fuzzy control rules

Fig.4 Structure of the fuzzy PID controller

Take the yaw rate and side slip angle as input variables, the definition of the input domain range of output variables are $[-8,8]$, the corresponding fuzzy domain for $[-1,1]$, fuzzy subset for $\{\mathrm{NB}$, 
NM, NS, ZE, PS, PM, PB \}, representing the \{negative big, negative medium, negative small, zero, positive small, positive medium, positive big\}.Combining with practical experience, the fuzzy rules table specific parameter setting[10],shown in table 1-3.

Tab.1 Fuzzy control rule of $\Delta K_{p}$

\begin{tabular}{llllllll}
\hline \multirow{2}{*}{$E$} & \multicolumn{7}{c}{$E C$} \\
\cline { 2 - 7 } & NB & NM & NS & ZO & PS & PM & PB \\
\hline NB & PB & PB & PM & PM & PS & ZO & ZO \\
NM & PB & PM & PM & PS & PS & ZO & NS \\
NS & PM & PM & PS & PS & ZO & NS & NS \\
ZO & PM & PM & PS & ZO & NS & NM & NM \\
PS & PS & PS & ZO & NS & NS & NM & NM \\
PM & PS & ZO & NS & NS & NM & NM & NB \\
PB & ZO & ZO & NM & NM & NM & NB & NB \\
\hline
\end{tabular}

Tab.2 Fuzzy control rule of $\Delta K_{i}$

\begin{tabular}{cccccccc}
\hline \multirow{2}{*}{$E$} & \multicolumn{7}{c}{$E C$} \\
\cline { 2 - 8 } & NB & NM & NS & ZO & PS & PM & PB \\
\hline NB & NB & NB & NM & NM & NS & ZO & ZO \\
NM & NB & NM & NM & NS & NS & ZO & ZO \\
NS & NB & NM & NS & NS & ZO & PS & PS \\
ZO & NM & NS & NS & ZO & PS & PM & PM \\
PS & NM & NS & ZO & PS & PS & PM & PB \\
PM & ZO & ZO & PS & PS & PM & PB & PB \\
PB & ZO & ZO & PS & PM & PM & PB & PB \\
\hline
\end{tabular}

Tab.3 Fuzzy control rule of $\Delta K_{d}$

\begin{tabular}{cccccccc}
\hline \multirow{2}{*}{$E$} & \multicolumn{7}{c}{$E C$} \\
\cline { 2 - 8 } & NB & NM & NS & ZO & PS & PM & PB \\
\hline NB & PS & NM & NM & NB & NB & NM & PS \\
NM & PS & NM & NB & NM & NM & NS & ZO \\
NS & ZO & NS & NM & NM & NS & NS & ZO \\
ZO & ZO & NS & NS & NS & NS & NS & ZO \\
PS & ZO & ZO & ZO & ZO & ZO & ZO & ZO \\
PM & PB & NS & PS & PS & PS & PS & PB \\
PB & PB & PM & PM & PM & PS & PS & PB \\
\hline
\end{tabular}

\section{Simulation analysis}

\section{Simulation diagram}

As actuator of ESP, the dynamic characteristics of hydraulic system must meet the requirements of the vehicle braking performance [11]. Using AMESim andMatlab joint simulation model of ESP hydraulic system, can not only play an AMESim simulation efficiency of prominent fluid machinery and with the aid of Matlab powerful numerical processing capabilities, complement each other, get more perfect complementary effect, structure as shown in figure 5.

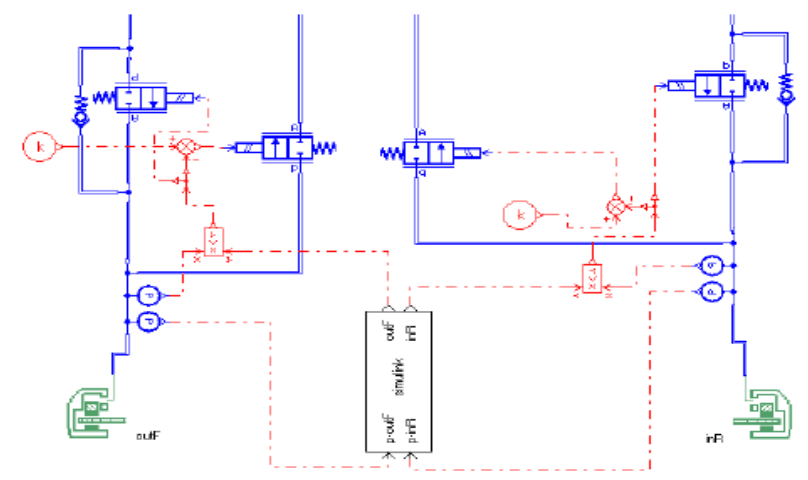

Fig.5 Co-simulation Frame Figure based on AMESim

\section{Results analysis}

In order to study the braking effect of universality, simulation analysis was different on the road friction coefficient. A low friction coefficient of 0.35 , a high friction coefficient is 0.8 , the vehicle speed before braking are $110 \mathrm{~km} / \mathrm{h}$, the maximum angle of the front wheel does not exceed $6.5^{\circ}$, the simulation time is 30 seconds, in a sinusoidal curve as the input to the vehicle braking when the yaw rate and sideslip angle as the observations, in order to verify the control effect, the ESP Fuzzy Control and uncontrolled contrast, the simulation results shown in Figure 6-9. 


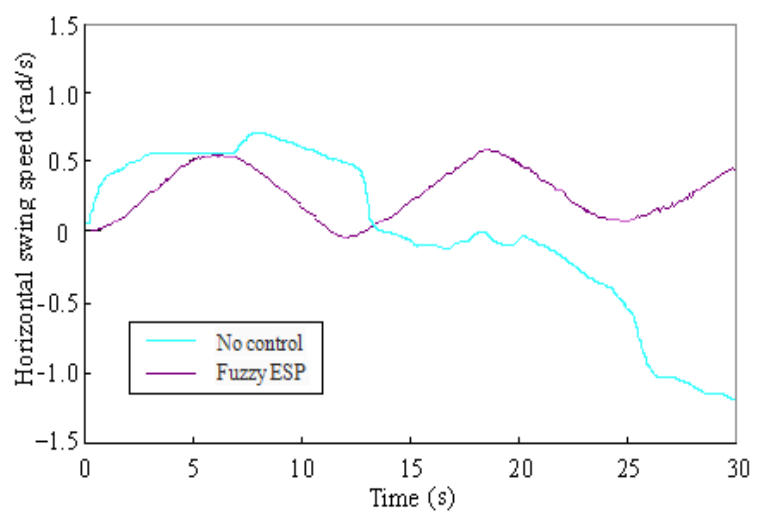

Fig.6 High friction coefficient, yaw rate curve

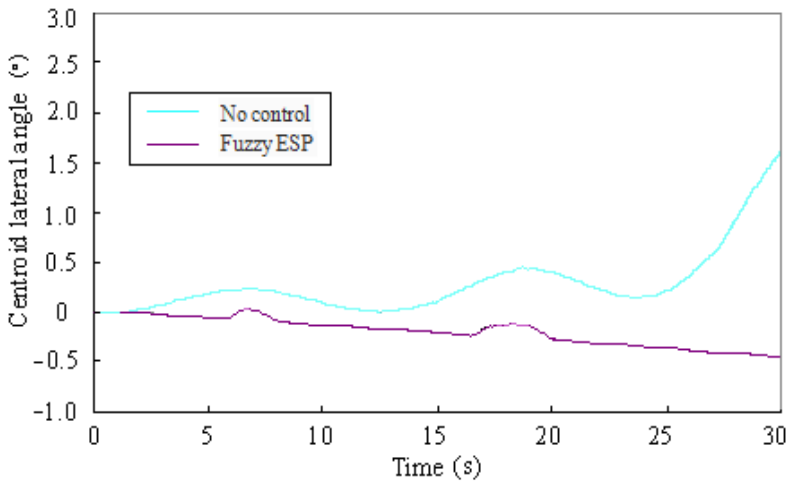

Fig.7 High adhesion coefficient, sideslip angle curve

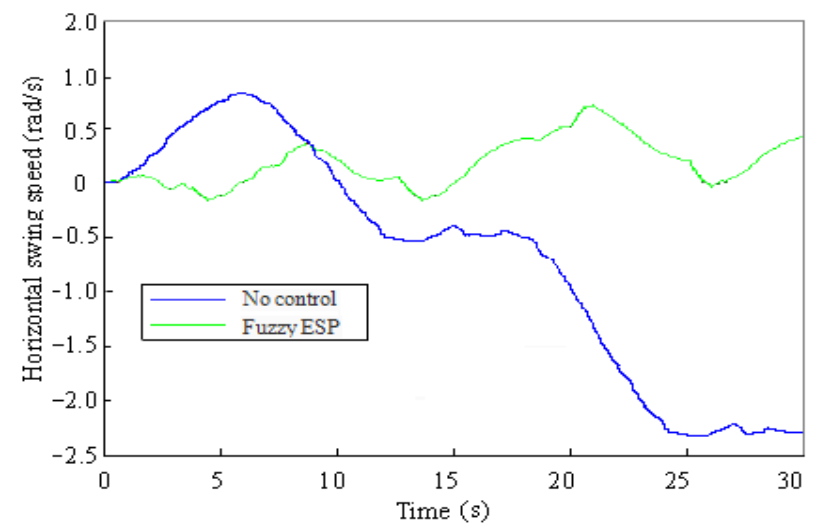

Fig.8 Low friction coefficient, yaw rate curve

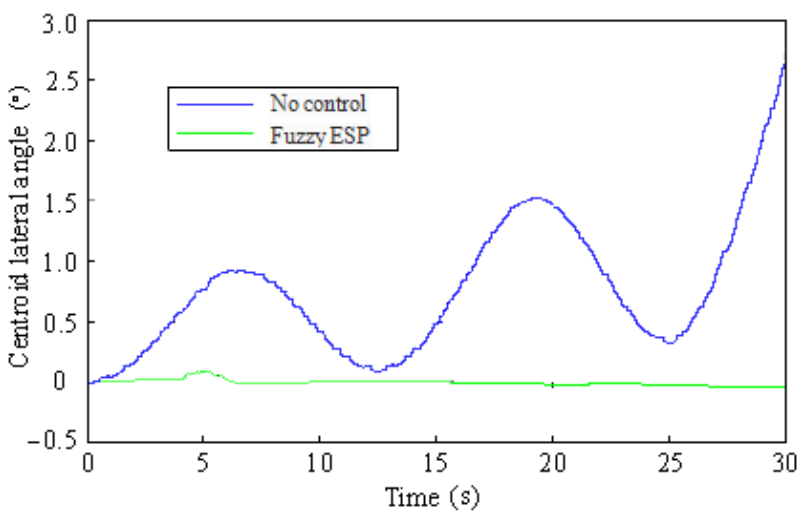

Fig.9 Low friction coefficient, sideslip angle curve

The results show that car at high speed emergency braking, the car will produce instability phenomenon, in the absence of stability control, the car instability phenomenon obviously, and there is a big risk. ESP uses fuzzy PID control car, has good stability, good control effect. 


\section{Conclusion}

Cars in complex conditions prone to instability when braking phenomenon, resulting in a significant impact on traffic safety, it is necessary to control the vehicle braking stability. This paper focuses on automobile ESP hydraulic system, analyzes the factors that affect vehiclebraking stability; the establishment of a AMESim hydraulic system model, and the cross-car braking yaw rate and sideslip angle as the study variables in the MATLAB environment under the joint simulation; analysis results show that the control method used car can effectively solve the problem of instability when braking, vehicle stability to study provide some reference.

\section{References}

[1] Taehyum Shim and Donald Margolis. Using mu Feed forward for Vehicle StabilityEnhancements [J]. Vehicle System Dynamics, 2001, 35(2): 103-119.

[2] Aleksander $\mathrm{Hac}$ and Mark O.Boide.Improvements in Vehicle Handling through IntegratedControl of Chassis System [J]. Int.J.Of Vehicle Design, 2002, 29(1): 23-50.

[3] H.Cherouat. Vehicle lateral motion control using lateral acceleration feedback[C]. Proceeding ofthe American Control Conference (ACC)2006

[4] Motoki Shino, Masao Nagai.Yaw-moment Control of Electric for Improving Handling andStability. JSAE Review, 2001, 22: 473-480.

[5] Masato Abe, Yoshio Kano, Kazuasa Suzuki, Yasuji Shibahata, Yoshimi Furukawa.Side-slip Control to Stabilize Vehicle Lateral Motion by Direct Yaw Control [J]. JSAE Review2001, 22: 413-419.

[6] Junjie He, David A Crolla. Coordination of active steering, driveline, and braking for integrated vehicle dynamics control [J]. Automobile Engineering, 2006 (220).

[7] Motoki Shino, Pongsathorn Raksincharoensak, Masao Nagai. Vehicle Handling and Stability Control by Integrated Control of Direct Yaw Moment and Active Steering[C]. Proceeding of theAdvanced Vehicle Control (AVEC) 2002

[8] Youssef A.Ghoneim, William C.lin, David M.Sidlosky, Hsien H.Chen, Yuen-Kwok Chin andMichael J.Tedrake. Integrated Chassis Control System to Enhance Vehicle Stability [J].International Journal of Vehicle Design, 2000, 23(1): 124-144.

[9] Masao Nagai, Yutaka Hirano and Sachiko Yamanaka. Integrated Control of Active Rear WheelSteering and Direct Yaw Moment Control [J]. Vehicle System Dynamics, 1997, 27: 357-370.

[10]Ansgar Trachtler. Integrated Vehicle Dynamics Control Using Active Brake, Steeringand Suspension Systems [J].International Journal of Vehicle Design,2004, 36(1).

[11]Aldo Sorniotti and Mauro Velardocchia.Hardware-In-the-Loop (HIL) Testing of ESP(Electronic Stability Program) Commercial Hydraulic Units and Implementation of NewControl Strategies. SAE paper 2004-01-2770. 